\title{
Quelle image « 3D » pour quelle application diagnostique et/ou thérapeutique en Chirurgie buccale?
}

\author{
Coudert $\mathrm{JL}^{1}$, Trunde $\mathrm{F}^{1}$, Bossard $\mathrm{D}^{2}$, Fortin $\mathrm{T}^{1}$ \\ ${ }^{1}$ Service de Consultations et de Traitements dentaires, Hospices Civils, Lyon, France \\ ${ }^{2}$ Hôpital Mermoz, Lyon, France \\ jean-loup.coudert@chu-lyon.fr
}

L'image radiologique conventionnelle est la projection d'un volume sur un plan, ce qui conduit à avec des superpositions des structures anatomiques. L'image obtenue est à 2 dimensions (2D) normée sur les axes $\mathrm{x}$ et $\mathrm{y}$. Elle ne possède pas de contenu informatif dans la $3^{\text {ème }}$ dimension.

La tomodensitométrie (Scanner X, Cone Beam et l'IRM) apportent des informations dans la $3^{\text {ème }}$ dimension de l'espace anatomique sous forme d'une pile de coupes. Chaque coupe est une image 2D normée en $\mathrm{x}$, y référencée sur l'axe $\mathrm{z}$ qui apporte la $3^{\text {ème }}$ dimension.

Comment exploiter l'information tridimensionnelle contenue dans la pile de coupes ?

Par des techniques bidimensionnelles : création d'images 2D dans d'autres plans que celui des coupes d'acquisition (plan coronal transverse). Ces images 2D peuvent être successives ou non, frontales ou sagittales, parallèles ou perpendiculaires à la crête maxillaire ou mandibulaire.

Par la reconstruction tridimensionnelle (3D) : elle présente une image vue sur un support (écran, papier, etc). Elle montre un volume anatomique virtuel dont les dimensions correspondent au volume réel : expression de la réalité sans déformation ni interprétation. Deux techniques principales sont proposées aux odontologistes : le rendu volumique et le rendu surfacique. Le rendu volumique est la technique la plus utilisée dans les logiciels proposés actuellement pour les implants. Il présente dans certaines situations anatomiques des superpositions trompeuses. Il ne nécessite que peu ou pas d'investissement temps machine et utilisateur. Le rendu surfacique est plus gourmand en temps machine et utilisateur car il nécessite deux étapes :

- la segmentation isole chacun des acteurs anatomiques souhaités par le clinicien pour ses besoins diagnostiques et thérapeutiques. On peut l'interpréter/la considérer comme une dissection mathématique dans les fichiers numériques des coupes d'acquisition, destinée à isoler un organe de ses voisins (une dent de l'os par exemple), par des techniques de traitement d'image comme le seuillage, la croissance de région, etc.

- la création de la scène : le rendu des surfaces des organes segmentés suit les mêmes règles que les images présentées dans les jeux vidéo ou les images de synthèse.

L'utilisation de la scène, pour préciser le diagnostic et prévoir le geste thérapeutique, se fait à 2 niveaux :

- qualitatif, subjectif : observer le bloc anatomique sous tous les angles d'intérêt, en rendant transparents ou invisibles des acteurs ; c'est une dissection virtuelle individualisée pour chaque patient.

- quantitatif en mesurant des distances entre les objets anatomiques d'intérêt, voire en déplaçant un objet (simulation, planification d'intervention) pour mesurer les conséquences éventuelles de l'intervention/du geste thérapeutique.

Ces deux qualités qualitatives et quantitatives du rendu surfacique permettent de limiter l'intervention au strict minimum donnant accès à une "chirurgie minimale invasive " car l'intervention peut être réalisée dans le virtuel puis les dimensions/informations quantitatives transférées dans le réel. 